\title{
CAUCHY PROBLEM FOR HYPERBOLIC EQUATIONS OF THIRD ORDER WITH VARIABLE EXPONENT OF NONLINEARITY
}

\author{
BuHRII O.M. ${ }^{1}$, KHOLYAVKA O.T. ${ }^{2}$, PUKACH P.YA. ${ }^{3}$, VOVK M.I. ${ }^{3}$
}

We investigate weak solutions of the Cauchy problem for the third order hyperbolic equations with variable exponent of the nonlinearity. The problem is considered in some classes of functions namely in Lebesgue spaces with variable exponents. The sufficient conditions of the existence and uniqueness of the weak solutions to given problem are found.

Key words and phrases: nonlinear hyperbolic equation, Cauchy problem, variable exponent of nonlinearity, Lebesgue space with variable exponent.

\footnotetext{
${ }^{1}$ Ivan Franko National University of Lviv, 1 Universytetska str., 79000, Lviv, Ukraine

2 Pidstryhach Institute for Applied Problems of Mechanics and Mathematics, 3b Naukova str., 79060, Lviv, Ukraine

${ }^{3}$ Lviv Polytechnic National University, 12 Bandera str., 79013, Lviv, Ukraine

E-mail: oleh.buhrii@lnu.edu.ua (Buhrii O.M.), oksana.t.kholyavka@gmail.com(Kholyavka O.T.), petro.y.pukach@lpnu.ua (Pukach P.Ya.), mira.i.kopych@gmail.com(Vovk M.I.)
}

\section{INTRODUCTION}

Let $n \in \mathbb{N}$ and $T>0$ be fixed numbers, $Q_{T}:=\mathbb{R}^{n} \times(0, T), Q_{t_{1}, t_{2}}:=\mathbb{R}^{n} \times\left(t_{1}, t_{2}\right), 0 \leq t_{1}<$ $t_{2} \leq T, B^{R}:=\left\{x \in \mathbb{R}^{n}|| x \mid<R\right\}, Q_{T}^{R}:=B^{R} \times(0, T), R>1$. In this study, we seek a weak solution $u: Q_{T} \rightarrow \mathbb{R}$ of the Cauchy problem

$$
\begin{gathered}
u_{t t}-\sum_{i, j=1}^{n}\left(a_{i j}(x, t) u_{x_{i}}\right)_{x_{j}}-\sum_{i, j=1}^{n}\left(b_{i j}(x, t) u_{x_{i}}\right)_{x_{j}}+\sum_{i=1}^{n} b_{i}(x, t) u_{x_{i}} \\
+c_{1}\left(x, t, u_{t}\right)+c_{2}(x, t) u=f_{0}(x, t)-\sum_{i=1}^{n}\left(f_{i}\right)_{x_{i}}(x, t) \text { in } Q_{T}, \\
\left.u\right|_{t=0}=u_{0}, \\
\left.u_{t}\right|_{t=0}=u_{1},
\end{gathered}
$$

where $a_{i j}, b_{i j}, b_{i}, c_{2}, f_{0}, f_{i}$ are some functions, $c_{1}\left(x, t, u_{t}\right)$ is a function on the type $\left|u_{t}\right|^{p(x)-2} u_{t}$, i.e., it is a nonlinear function with the variable exponent of the nonlinearity $p=p(x)$. Problems for the nonlinear PDEs with the variable exponents of the nonlinearity appear in many applications, such as fluid dynamics, nonlinear elasticity, etc. They are investigated in some special classes of the functions namely in the Lebesgue and Sobolev spaces with variable exponents (see $[3,16,42,43])$.

Let us consider the equation

$$
u_{t t}+A u_{t}+B u+\left|u_{t}\right|^{p(x)-2} u_{t}+|u|^{q(x)-2} u=0, \quad(x, t) \in \Omega \times(0, T),
$$

$\mathrm{y} \Delta \mathrm{K} 517.955 .2$

2010 Mathematics Subject Classification: 35L75, 35L30, 35D30. 
where $A$ and $B$ are some operators, $p(x), q(x)$ are some functions, $\Omega \subset \mathbb{R}^{n}$. In case $A=0$ and $B=-\triangle$ (here and below $\triangle$ denotes the Laplace operator), type (4) equations are considered in $[14,23,24,38]$. In particular, [23] is devoted to the existence results for the weak solution of the initial-boundary value problem for equation (4) in a bounded cylindrical domain. The existence of the global (in time) solutions $u$ to such problems and the behaviour of $u$ as $t \rightarrow+\infty$ are investigated in [38]. In [24], the authors consider the initial-boundary value problem for equation (4) in the domain unbounded in spatial variables. The existence and uniqueness of the problem's solution is proved without any restrictions on solution behavior and the initial data as $|x| \rightarrow+\infty$. The hyperbolic variational inequalities of the second order that correspond to the equation (4) with $p(x) \equiv 2$ and $q(x)>1$ are studied in [14].

In case $A=B=-\triangle$, type (4) equations are considered in [22,26,37]. In particular, the behaviour as $t \rightarrow+\infty$ of the global solutions to the initial-boundary problems for hyperbolic equations of the third order are obtained in [37]. The corresponding hyperbolic variational inequalities in bounded and unbounded domain with the spatial variables are studied in [22] and [26] respectively if $p(x)>1$ and $q(x) \equiv 2$. The existence and uniqueness theorems are proved. In the case of the unbounded domain $\Omega$, the results are obtained without any restrictions on behavior (as $|x| \rightarrow+\infty$ ) of the solutions and data-in.

In [13] and [17], the authors study the initial-boundary value problems for equation (4) with

$$
A u_{t}=-\operatorname{div}\left(\left|\nabla u_{t}\right|^{r(x)-2} \nabla u_{t}\right), \quad B u=-\triangle u,
$$

$q(x) \equiv 2, r(x)>2$, and $p(x)>1$. The existence and uniqueness of the problem's solution are proved for the bounded domain $\Omega$. If

$$
A u_{t}=-\operatorname{div}\left(\left|u_{t}\right|^{s-2} \nabla u_{t}\right) \quad(s>2), \quad B u=-\Delta u,
$$

$q(x) \equiv 2$, and $p(x)>2$, then the initial-boundary value problem for equation (4) with the unbounded domain $\Omega$ is investigated in [36]. Conditions of existence of the problem's solution are obtained without any restrictions on the behavior (as $|x| \rightarrow+\infty$ ) of the solutions and data-in.

The various problems for the hyperbolic equations, hyperbolic-parabolic systems, parabolic and elliptic equations with variable exponents of nonlinearity have also studied in $[2-8,10$ $12,19-21,25,27,31,32,34,35,39]$ etc. The application is given in [40,41,43].

Notice that, if some additional conditions are satisfied, then (1) coincides with (4), where $A=B=-\triangle, 1<p(x)<2$, and $q(x) \equiv 2$. We prove the existence and uniqueness of the solutions to problem (1)-(3) in classes of the functions with some behaviour as $|x| \rightarrow+\infty$ if coefficients of the equation (1) satisfy some growing conditions.

The paper is organized as follows. In Section 1, we formulate the considered problem and main results. The auxiliary statements are given in Section 2. Finally, in Section 3 we prove the main statements.

\section{NOTATION AND STATEMENT OF MAIN RESUlT}

Let $\|\cdot\|_{B} \equiv\|\cdot ; B\|$ is a norm of some Banach space $B, B^{*}$ is a dual space, and $\langle\cdot, \cdot\rangle_{B}$ is a scalar product between $B^{*}$ and $B$. The notation $X+Y$ means the sum of the Banach spaces $X$ and $Y$ (see [18, p. 23] for more details). 
Suppose that $m \in \mathbb{N}, \mathfrak{p} \in[1, \infty], X$ is a Banach space, $\mathcal{O}=\Omega$ or $\mathcal{O}=Q_{T}, \mathcal{M}(\mathcal{O})$ is a set of all measurable functions $v: \mathcal{O} \rightarrow \mathbb{R}, C^{m}(\mathcal{O}), C_{0}(\mathcal{O})$, and $D(\mathcal{O})$ are the spaces of the smooth functions (see [1, p. 9, 19]), $C_{0}^{m}(\mathcal{O}):=C^{m}(\mathcal{O}) \cap C_{0}(\mathcal{O}), L^{\mathfrak{p}}(\mathcal{O})$ is the Lebesgue space (see [1, p. 22, 24]), $W^{m, \mathfrak{p}}(\mathcal{O})$ and $W_{0}^{m, \mathfrak{p}}(\mathcal{O})$ are the Sobolev spaces (see $\left[1\right.$, p. 45]), $H^{m}(\mathcal{O}):=W^{m, 2}(\mathcal{O})$, $H_{0}^{m}(\mathcal{O}):=W_{0}^{m, 2}(\mathcal{O}), C([0, T] ; X)$ and $C^{m}([0, T] ; X)$ are the spaces of the $X$-valued smooth functions defined on $[0, T]$ (see [18, p. 147]), $L^{\mathfrak{p}}(0, T ; X)$ is the Lebesgue-Bochner space (see [18, p. 155]). Also suppose that $\mathcal{B}_{+}(\mathcal{O}):=\left\{\mathfrak{q} \in L^{\infty}(\mathcal{O}) \quad \mid \quad \underset{y \in \mathcal{O}}{\text { ess }} \inf \mathfrak{q}(y)>0\right\}$. For the sake of convenience, we will write $u(t)$ instead of $u(\cdot, t)$ and $L^{p}(0, T)$ instead of $L^{p}((0, T))$ etc. For every $\mathfrak{q} \in \mathcal{B}_{+}(\mathcal{O})$ by definition, put

$$
\begin{gathered}
\mathfrak{q}_{0}:=\underset{y \in \mathcal{O}}{\operatorname{essinf}} \mathfrak{q}(y), \quad \mathfrak{q}^{0}:=\underset{y \in \mathcal{O}}{\operatorname{ess} \sup } \mathfrak{q}(y), \\
\rho_{\mathfrak{q}}(v ; \mathcal{O}):=\int_{\mathcal{O}}|v(y)|^{\mathfrak{q}(y)} d y, \quad v \in \mathcal{M}(\mathcal{O}), \\
\mathfrak{q}^{\prime}(y):=\frac{\mathfrak{q}(y)}{\mathfrak{q}(y)-1} \text { for a.e. } y \in \mathcal{O}
\end{gathered}
$$

(note that $\frac{1}{\mathfrak{q}(y)}+\frac{1}{\mathfrak{q}^{\prime}(y)}=1$ for a.e. $y \in \mathcal{O}$ and $\mathfrak{q}^{\prime} \in \mathcal{B}_{+}(\mathcal{O})$, if $\mathfrak{q}_{0}>1$ ).

Assume that $\mathfrak{q} \in \mathcal{B}_{+}(\mathcal{O})$ and $\mathfrak{q}_{0}>1$. The set $L^{\mathfrak{q}(y)}(\mathcal{O}):=\left\{v \in \mathcal{M}(\mathcal{O}) \mid \rho_{\mathfrak{q}}(v ; \mathcal{O})<+\infty\right\}$ with the Luxemburg norm $\left\|v ; L^{\mathfrak{q}(y)}(\mathcal{O})\right\|:=\inf \left\{\lambda>0 \mid \rho_{\mathfrak{q}}(v / \lambda ; \mathcal{O}) \leq 1\right\}$ is called a Lebesgue space with variable exponent. By definition, put $L_{\text {loc }}^{\mathfrak{q}(y)}(\mathcal{O}):=\{u \in \mathcal{M}(\mathcal{O}) \mid \forall$ bounded $\left.G \Subset \mathcal{O}: u \in L^{\mathfrak{q}(y)}(G)\right\}$. In the similar way, we define the spaces $L_{\text {loc }}^{\infty}(\mathcal{O}), H_{\text {loc }}^{1}(\mathcal{O})$, etc. Suppose that the following conditions are satisfied.

(A): $a_{i j} \in L^{\infty}\left(0, T ; L_{\mathrm{loc}}^{\infty}\left(\mathbb{R}^{n}\right)\right), a_{i j}=a_{j i}(i, j=\overline{1, n})$;

$\sum_{i, j=1}^{n} a_{i j}(x, t) \xi_{i} \xi_{j} \geq a_{0}|\xi|^{2}$ for all $\xi \in \mathbb{R}^{n}$ and for a.e. $(x, t) \in Q_{T}$, where $a_{0}>0$;

$\forall R_{1}>1$ ess sup $\left|a_{i j}(x, t)\right| \leq \alpha R_{1}^{\theta}$, where $\theta \in[0,1)$ and $\alpha>0$;

$$
Q_{T}^{R_{1}}
$$

(B): $b_{i j},\left(b_{i j}\right)_{t} \in L^{\infty}\left(0, T ; L_{\mathrm{loc}}^{\infty}\left(\mathbb{R}^{n}\right)\right), b_{i j}=b_{j i}(i, j=\overline{1, n}) ; b_{i} \in L^{\infty}\left(Q_{T}\right)(i=\overline{1, n})$;

$\sum_{i, j=1}^{n} b_{i j}(x, t) \xi_{i} \xi_{j} \geq b_{0}|\xi|^{2}$ and $\sum_{i, j=1}^{n}\left(b_{i j}\right)_{t}(x, t) \xi_{i} \xi_{j} \leq 0$ for all $\xi \in \mathbb{R}^{n}$ and for a.e. $(x, t) \in Q_{T}$, where $b_{0}>0$;

$\forall R_{2}>1$ ess sup $\left|b_{i j}(x, t)\right| \leq \beta R_{2}^{\theta}$, where $\theta$ is taken from condition (A) and $\beta>0$;

$$
Q_{T}^{R_{2}}
$$

(C): $c_{1}(\cdot, \cdot, \xi)$ is a measurable function on $Q_{T}$ for all $\xi \in \mathbb{R} ; c_{1}(x, t, \cdot)$ is a continuous function on $\mathbb{R}$ for a.e. $(x, t) \in Q_{T} ; c_{2}, c_{2, t} \in L^{\infty}\left(0, T ; L_{\text {loc }}^{\infty}\left(\mathbb{R}^{n}\right)\right)$;

$\left(c_{1}(x, t, \xi)-c_{1}(x, t, \eta)\right)(\xi-\eta) \geq 0, c_{1}(x, t, \xi) \xi \geq c_{1,0}|\xi|^{p(x)},\left|c_{1}(x, t, \xi)\right| \leq c_{1}^{0}|\xi|^{p(x)-1}$ for all $\xi, \eta \in \mathbb{R}$ and for a.e. $(x, t) \in Q_{T}$, where $c_{1,0} \in \mathbb{R}, c_{1}^{0}>0, p \in \mathcal{B}_{+}(\Omega)$, and $1<p_{0} \leq p^{0}<2$;

$0<c_{2,0} \leq c_{2}(x, t) \leq c_{2}^{0},\left(c_{2, t}\right)_{0} \leq c_{2, t}(x, t) \leq\left(c_{2, t}\right)^{0} \leq 0$ for all $R_{3}>1$ and for a.e. $(x, t) \in Q_{T}^{R_{3}}$, where constants $c_{2,0}, c_{2}^{0},\left(c_{2, t}\right)_{0}$, and $\left(c_{2, t}\right)^{0}$ depend on $R_{3}$;

(F): $f_{0}, f_{1}, \ldots, f_{n} \in L^{2}\left(0, T ; L_{\text {loc }}^{2}\left(\mathbb{R}^{n}\right)\right)$;

(U): $u_{0} \in H_{\text {loc }}^{1}\left(\mathbb{R}^{n}\right), u_{1} \in L_{\text {loc }}^{2}\left(\mathbb{R}^{n}\right)$. 
Definition 1. A real-valued function $u$ is called a weak solution to problem (1)-(3) if $u \in L^{\infty}\left(0, T ; H_{l o c}^{1}\left(\mathbb{R}^{n}\right)\right) \cap C\left([0, T] ; L_{\text {loc }}^{2}\left(\mathbb{R}^{n}\right)\right), u_{t} \in L^{2}\left(0, T ; H_{l o c}^{1}\left(\mathbb{R}^{n}\right)\right) \cap L_{l o c}^{p(x)}\left(\bar{Q}_{T}\right), u$ satisfies condition (2), and for all functions $v \in L^{2}\left(0, T ; H_{l o c}^{1}\left(\mathbb{R}^{n}\right)\right) \cap L_{l o c}^{p(x)}\left(\bar{Q}_{T}\right)$ such that $v_{t} \in L_{l o c}^{2}\left(\bar{Q}_{T}\right)$, for any $\tau \in(0, T]$, for arbitrary $\varphi \in C_{0}^{1}\left(\mathbb{R}^{n}\right)$, the following equality is true:

$$
\begin{aligned}
\int_{\mathbb{R}^{n}} u_{t}(x, \tau) v(x, \tau) \varphi(x) d x+\int_{Q_{\tau}}\left[-u_{t} v_{t} \varphi+\sum_{i, j=1}^{n} a_{i j}(x, t) u_{x_{i} t}(v \varphi)_{x_{j}}\right. \\
\left.+\sum_{i, j=1}^{n} b_{i j}(x, t) u_{x_{i}}(v \varphi)_{x_{j}}+\sum_{i=1}^{n} b_{i}(x, t) u_{x_{i}} v \varphi+c_{1}\left(x, t, u_{t}\right) v \varphi+c_{2}(x, t) u v \varphi\right] d x d t \\
=\int_{\mathbb{R}^{n}} u_{1}(x) v(x, 0) \varphi(x) d x+\int_{Q_{\tau}}\left[f_{0}(x, t) v \varphi+\sum_{i=1}^{n} f_{i}(x, t)(v \varphi)_{x_{i}}\right] d x d t .
\end{aligned}
$$

The main results of our paper are next theorems.

Theorem 1 (the uniqueness). Suppose that conditions (A)-(U) hold. Then problem (1)-(3) has at most one solution in the class of functions $u$, which for any $R \geq 1$ satisfy the condition

$$
\int_{Q_{T}^{R}}\left|u_{t}(x, t)\right|^{2} d x d t \leq a \exp \left(b R^{2(1-\theta)}\right),
$$

where $a$ and $b$ are nonnegative constants and $\theta$ is taken from conditions $(\boldsymbol{A})$ and $(\boldsymbol{B})$.

Theorem 2 (the existence). If conditions $(\boldsymbol{A})-(\boldsymbol{U})$ hold and for all $R>1$ :

$$
\int_{B^{R}}\left[\left|u_{1}(x)\right|^{2}+R^{\theta} \sum_{i=1}^{n}\left|u_{0, x_{i}}(x)\right|^{2}\right] d x+\int_{Q_{T}^{R}} \sum_{i=0}^{n}\left|f_{i}(x, t)\right|^{2} d x d t \leq a \exp \left(b R^{2(1-\theta)}\right),
$$

where $a$ and $b$ are nonnegative constants, then problem (1)-(3) has a weak solution.

\section{AUXILIARY FACTS}

Let us consider the function $\zeta \in C^{2}(\mathbb{R})$ such that $\zeta(\xi)=\left\{\begin{array}{ll}1, & \xi \leq 0, \\ 0, & \xi \geq 1,\end{array}\right.$ and $0 \leq \zeta(\xi) \leq 1$ for $\xi \in \mathbb{R}$. By definition, we put

$$
h_{R, \varkappa}(x)=\zeta\left(\frac{|x|-R}{\varkappa}\right), \quad \varphi(x)=\left|h_{R, \varkappa}(x)\right|^{\gamma},
$$

where $R>1, \varkappa>0$, and $\gamma>2$.

The following lemmas are needed for the sequel.

Lemma 1. Take an arbitrary $j \in\{1,2, \ldots, n\}$. Then the following statements are true:

1) $0 \leq h_{R, \varkappa} \leq 1$ and there exists a constant $\widehat{h}>0$ such that $\left|\frac{\partial h_{R, \varkappa}(x)}{\partial x_{j}}\right| \leq \frac{\widehat{h}}{\varkappa}$;

2) foll all $x \in \mathbb{R}^{n}$ we obtain

$$
\varphi(x)=1 \text { if }|x| \leq R \text { and } \varphi(x)=0 \text { if }|x| \geq R+\varkappa \text {; }
$$


3) if $|x|<R+\varkappa$, then $\left|\varphi_{x_{j}}(x)\right| \leq \frac{\gamma \widehat{h}}{\varkappa}\left|h_{R, \varkappa}(x)\right|^{\gamma-1}$;

4) if $|x|<R+\varkappa$, then the following inequality is true:

$$
\frac{\left|\varphi_{x_{j}}(x)\right|^{2}}{\varphi(x)} \leq\left(\frac{\gamma \widehat{h}}{\varkappa}\right)^{2}\left|h_{R, \varkappa}(x)\right|^{\gamma-2} .
$$

Proof. The first estimate from 1) and equality from 2) are obvious. The second estimate from 1) inferred from the equality $\left|\frac{\partial h_{R, \varkappa}(x)}{\partial x_{j}}\right|=\left|\zeta^{\prime} \frac{1}{\varkappa} \frac{x_{j}}{x \mid}\right|$. To prove the statement from 3$)$ we need to use the equality $\varphi_{x_{j}}(x)=\gamma\left|h_{R, \varkappa}(x)\right|^{\gamma-1} \frac{\partial h_{R, \varkappa}(x)}{\partial x_{j}}$.

It is clear that from result of 3 ), we get the estimate

$$
\frac{\left|\varphi_{x_{j}}(x)\right|^{2}}{\varphi(x)} \leq\left(\frac{\gamma \widehat{h}}{\varkappa}\right)^{2} \frac{\left|h_{R, \varkappa}(x)\right|^{2(\gamma-1)}}{\left|h_{R, \varkappa}(x)\right|^{\gamma}} \leq\left(\frac{\gamma \widehat{h}}{\varkappa}\right)^{2}\left|h_{R, \varkappa}(x)\right|^{\gamma-2},
$$

and (12) holds. Thus, Lemma 1 is proved.

Lemma 2. Suppose that $\Omega$ is a bounded domain in $\mathbb{R}^{n}$ with the piecewise smooth boundary, $r \in L^{\infty}(\Omega), r>1$. Then for all functions $w \in L^{2}\left(0, T ; H_{0}^{1}(\Omega)\right) \cap L^{r(x)}\left(Q_{T}\right) \cap L^{\infty}\left(0, T ; L^{2}(\Omega)\right)$ such that $w_{t} \in L^{2}\left(0, T ; H^{-1}(\Omega)\right)+L^{r^{\prime}(x)}\left(Q_{T}\right)$ and for any $s, \tau \in[0, T]$ such that $s<\tau$, the following formula of integration by parts is correct:

$$
\int_{Q_{s, \tau}} w_{t}(x, t) w(x, t) d x d t=\frac{1}{2} \int_{\Omega_{\tau}}|w(x, \tau)|^{2} d x-\frac{1}{2} \int_{\Omega_{s}}|w(x, s)|^{2} d x .
$$

Proof. In [13], formula (13) is proved for a.e. $s, \tau \in[0, T]$ such that $s<\tau$. From conditions of Lemma 2, we get $w \in C\left([0, T] ; H^{-1}(\Omega)+L^{r^{\prime}(x)}(\Omega)\right)$. Then, from [29], it follows that $w \in C_{w}\left([0, T] ; L^{2}(\Omega)\right)$. For any $s, \tau \in[0, T]$, let us extends the function $w$ in regard of its continuity outside the interval $[s, \tau]$ by constants and then, similarly as in [9] (see also Lemma 4.5 [3, p. 119]), we obtain (13). Lemma 2 is proved.

Remark 1. In view of (13), for every $\varphi \in C^{\infty}(\Omega)$ and $\psi \in C^{\infty}([0, T])$, we get

$$
\begin{aligned}
\int_{Q_{s, \tau}} & w_{t}(x, t) w(x, t) \varphi(x) \psi(t) d x d t=\frac{1}{2} \int_{\Omega}|w(x, \tau)|^{2} \varphi(x) \psi(\tau) d x \\
- & \frac{1}{2} \int_{\Omega}|w(x, s)|^{2} \varphi(x) \psi(s) d x-\frac{1}{2} \int_{Q_{s, \tau}}|w(x, t)|^{2} \varphi(x) \psi_{t}(t) d x d t .
\end{aligned}
$$

Proposition 1 ([33], part IV, §2, Theorems 7-8). If $n, k \in \mathbb{N}, G$ is a bounded domain in $\mathbb{R}^{n}$, $\partial G \subset C^{k}$, and $k \geq\left[\frac{n}{2}\right]+2$, then there exist a sequence $\left\{\lambda_{j}\right\}_{j \in \mathbb{N}}$ of the positive numbers and a sequence $\left\{w^{j}\right\}_{j \in \mathbb{N}} \subset H^{k}(G) \cap C^{1}(\bar{G})$ of the linearly independent functions whose finite linear combinations are dense in the space $H^{2}(G) \cap H_{0}^{1}(G)$, and we have

$$
-\Delta w^{j}=\lambda_{j} w^{j} \quad \text { in } \quad G \quad \text { and }\left.\quad w^{j}\right|_{\partial \Omega}=0 \quad \text { for every } \quad j \in \mathbb{N} \text {. }
$$


Proposition 2 ([12], Lemma 1). If $\mathfrak{q} \in \mathcal{B}_{+}(\mathcal{O})$ and $\mathfrak{q}_{0}>1$ (see (5)), then for every $a, b \in \mathbb{R}$, for every $\eta>0$, and for a.e. $y \in \mathcal{O}$ the generalized Young inequality

$$
a b \leq \eta|a|^{\mathfrak{q}(y)}+Y_{\mathfrak{q}}(\eta)|b|^{\mathfrak{q}^{\prime}(y)}
$$

holds. Here, $Y_{\mathfrak{q}}(\eta)=\left(\mathfrak{q}^{0}-1\right) \mathfrak{q}_{0}^{-\mathfrak{q}_{0} /\left(\mathfrak{q}_{0}-1\right)} \eta^{-1 /\left(\mathfrak{q}_{0}-1\right)}, \mathfrak{q}^{0}$ is taken from (5), $\mathfrak{q}^{\prime}$ is taken from (7) and if $\mathfrak{q} \equiv 2$, then $Y_{2}(\eta)=1 /(4 \eta)$.

For every $\mathfrak{q} \in \mathcal{B}_{+}(\mathcal{O})$, by definition, put $S_{\mathfrak{q}}(s):=\max \left\{s^{\mathfrak{q}_{0}}, \mathfrak{s}^{\mathfrak{q}^{0}}\right\}, s \geq 0$.

Proposition 3 ([10], p. 168, Lemma 1). Suppose that $\mathfrak{q} \in \mathcal{B}_{+}(\mathcal{O}), \mathfrak{q}_{0}>1, S_{\mathfrak{q}}$ is defined above, and $\rho_{\mathfrak{q}}$ is defined by (6). Then for every $v \in \mathcal{M}(\mathcal{O})$ the following statements are fulfilled:

i) $\left\|v ; L^{\mathfrak{q}(y)}(\mathcal{O})\right\| \leq S_{1 / \mathfrak{q}}\left(\rho_{\mathfrak{q}}(v ; \mathcal{O})\right)$ if $\rho_{\mathfrak{q}}(v ; \mathcal{O})<+\infty$;

ii) $\rho_{\mathfrak{q}}(v ; \mathcal{O}) \leq S_{\mathfrak{q}}\left(\left\|v ; L^{\mathfrak{q}(y)}(\mathcal{O})\right\|\right)$ if $\left\|v ; L^{\mathfrak{q}(y)}(\mathcal{O})\right\|<+\infty$.

Now we will prove the existence of the weak solution to problem (1)-(3). For this purpose, let us fix $R>1$ and consider the auxiliary problem in the bounded domain $Q_{T}^{R}=B^{R} \times(0, T)$ :

$$
\begin{gathered}
u_{t t}-\sum_{i, j=1}^{n}\left(a_{i j}(x, t) u_{x_{i} t}\right)_{x_{j}}-\sum_{i, j=1}^{n}\left(b_{i j}(x, t) u_{x_{i}}\right)_{x_{j}}+\sum_{i=1}^{n} b_{i}(x, t) u_{x_{i}} \\
+c_{1}\left(x, t, u_{t}\right)+c_{2}(x, t) u=f_{0}^{R}(x, t)-\sum_{i=1}^{n} f_{i, x_{i}}^{R}(x, t), \quad(x, t) \in Q_{T}^{R}, \\
u(x, 0)=u_{0}^{R}(x), \quad u_{t}(x, 0)=u_{1}^{R}(x), \quad x \in B^{R}, \\
\left.u\right|_{\partial B^{R} \times[0, T]}=0 .
\end{gathered}
$$

Here $f_{i}^{R}(x, t)=f_{i}(x, t) \chi^{R}(x)(i=\overline{0, n}) ; u_{0}^{R}(x)=u_{0}(x) \chi^{R}(x), u_{1}^{R}(x)=u_{1}(x) \chi^{R}(x),(x, t) \in Q_{T}$,

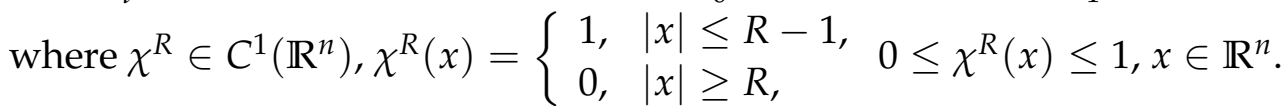

Definition 2. A real-valued function $u$ is called a weak solution to problem (16)-(18) if

$$
\begin{aligned}
u & \in L^{\infty}\left(0, T ; H_{0}^{1}\left(B^{R}\right)\right) \cap C\left([0, T] ; L^{2}\left(B^{R}\right)\right), \\
u_{t} & \in L^{2}\left(0, T ; H_{0}^{1}\left(B^{R}\right)\right) \cap L^{p(x)}\left(Q_{T}^{R}\right) \cap C\left([0, T] ; H^{-1}\left(B^{R}\right)+L^{p^{\prime}(x)}\left(B^{R}\right)\right),
\end{aligned}
$$

$u$ satisfies the condition $u^{R}(0)=u_{0}^{R}$ and the equality

$$
\begin{aligned}
& \int_{B^{R}} u_{t}^{R}(x, \tau) v(x, \tau) d x+\int_{Q_{\tau}^{R}}\left[-u_{t}^{R} v_{t}+\sum_{i, j=1}^{n} a_{i j}(x, t) u_{x_{i}}^{R} v_{x_{j}}+\sum_{i, j=1}^{n} b_{i j}(x, t) u_{x_{i}}^{R} v_{x_{j}}+\sum_{i=1}^{n} b_{i}(x, t) u_{x_{i}}^{R} v\right. \\
& \left.+c_{1}\left(x, t, u_{t}^{R}\right) v+c_{2}(x, t) u^{R} v\right] d x d t=\int_{B^{R}} u_{1}^{R}(x) v(x, 0) d x+\int_{Q_{\tau}^{R}}\left[f_{0}^{R}(x, t) v+\sum_{i=1}^{n} f_{i}^{R}(x, t) v_{x_{i}}\right] d x d t
\end{aligned}
$$

holds for every $\tau \in(0, T]$ and $v \in L^{2}\left(0, T ; H_{0}^{1}\left(B^{R}\right)\right) \cap L^{p(x)}\left(Q_{T}^{R}\right)$ such that $v_{t} \in L^{2}\left(Q_{T}^{R}\right)$.

Theorem 3. Suppose that conditions (A)-(U) are satisfied. Then problem (16)-(18) has a weak solution. 
Proof. We use the Faedo-Galerkin method. Let $\left\{w^{j}\right\}_{j \in \mathbb{N}}$ is taken from Proposition 1 with $G=B^{R}$. Without loss of generality we can assume that this sequence is orthonormal in $L^{2}\left(B^{R}\right)$. Then we will find the solution to problem (16)-(18) as a limit of the sequence of the functions $\left\{u^{N}\right\}_{N \in \mathbb{N}}$, where $u^{N}(x, t)=\sum_{l=1}^{N} C_{l}^{N}(t) w^{l}(x),(x, t) \in Q_{T}^{R}$. The functions $\left(C_{1}^{N}, \ldots, C_{N}^{N}\right)$ are defined from the Cauchy problem

$$
\begin{gathered}
\int_{B^{R}}\left[u_{t t}^{N} w^{k}+\sum_{i, j=1}^{n} a_{i j}(x, t) u_{x_{i}}^{N} w_{x_{j}}^{k}+\sum_{i, j=1}^{n} b_{i j}(x, t) u_{x_{i}}^{N} w_{x_{j}}^{k}+\sum_{i=1}^{n} b_{i}(x, t) u_{x_{i}}^{N} w^{k}\right. \\
\left.+c_{1}\left(x, t, u_{t}^{N}\right) w^{k}+c_{2}(x, t) u^{N} w^{k}\right] d x=\int_{B^{R}}\left[f_{0}^{R}(x, t) w^{k}+\sum_{i=1}^{n} f_{i}^{R}(x, t) w_{x_{i}}^{k}\right] d x, \\
C_{k}^{N}(0)=u_{0, k}^{N, R}, \quad C_{k, t}^{N}(0)=u_{1, k}^{N, R}, \quad k=\overline{1, N} .
\end{gathered}
$$

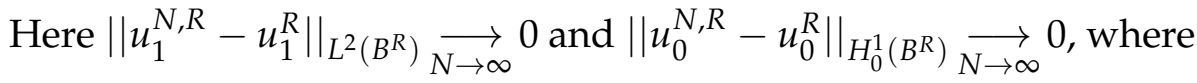

$$
u_{0}^{N, R}(x) \equiv \sum_{k=1}^{N} u_{0, k}^{N, R} w^{k}(x), \quad u_{1}^{N, R}(x) \equiv \sum_{k=1}^{N} u_{1, k}^{N, R} w^{k}(x), \quad x \in B^{R} .
$$

It is clear that $u^{N, R}(0)=u_{0}^{N, R}, \quad u_{t}^{N, R}(0)=u_{1}^{N, R}$.

According to the assumptions of Theorem 3 and by the Caratheodory Theorem (see. [15, p. 54]), we infer that there exists a solution to problem (19)-(20) which is determined in some interval $\left[0, t_{N}\right]$. From the estimates obtained below it follows that $t_{N}=T$.

Multiplying (19) by the functions $C_{k, t}^{N}$ respectively, summing by $k$ from 1 to $N$, and integrating along the interval $(0, \tau) \subset(0, T)$, we obtain

$$
\begin{aligned}
& \int_{Q_{\tau}^{R}}\left[u_{t t}^{N} u_{t}^{N}+\sum_{i, j=1}^{n} a_{i j}(x, t) u_{x_{i}}^{N} u_{x_{j} t}^{N}+\sum_{i, j=1}^{n} b_{i j}(x, t) u_{x_{i}}^{N} u_{t x_{j}}^{N}+\sum_{i=1}^{n} b_{i}(x, t) u_{x_{i}}^{N} u_{t}^{N}\right. \\
& \left.\quad+c_{1}\left(x, t, u_{t}^{N}\right) u_{t}^{N}+c_{2}(x, t) u^{N} u_{t}^{N}\right] d x d t=\int_{Q_{\tau}^{R}}\left[f_{0}^{R}(x, t) u_{t}^{N}+\sum_{i=1}^{n} f_{i}^{R}(x, t) u_{x_{i} t}^{N}\right] d x d t .
\end{aligned}
$$

Taking into account the conditions of Theorem 3, from equality (21) and Proposition 2 we get

$$
\begin{aligned}
& \int_{B^{R}}\left[\left|u_{t}^{N}(\tau)\right|^{2}+b_{0} \sum_{i=1}^{n}\left|u_{x_{i}}^{N}(\tau)\right|^{2}+c_{2,0}\left|u^{N}(\tau)\right|^{2}\right] d x \\
& \quad+\int_{Q_{\tau}^{R}}\left[\left(2 a_{0}-2 \delta_{0}\right) \sum_{i=1}^{n}\left|u_{x_{i}}^{N}\right|^{2}+2 c_{1,0}\left|u_{t}^{N}\right|^{p(x)}\right] d x d t \leq \int_{Q_{\tau}^{R}}\left[2\left|u_{t}^{N}\right|^{2}+\widetilde{B} \sum_{i=1}^{n}\left|u_{x_{i}}^{N}\right|^{2}\right] d x d t \\
& \quad+\int_{B^{R}}\left[\left|u_{1}^{N, R}\right|^{2}+b^{0} \sum_{i=1}^{n}\left|u_{0, x_{i}}^{N, R}\right|^{2}+c_{2}^{0}\left|u_{0}^{N, R}\right|^{2}\right] d x+\int_{Q_{\tau}^{R}}\left[\left|f_{0}^{R}\right|^{2}+2 Y_{2}\left(\delta_{0}\right) \sum_{i=1}^{n}\left|f_{i}^{R}\right|^{2}\right] d x d t,
\end{aligned}
$$

$\delta_{0}>0$, where $\widetilde{B}=\underset{(x, t) \in Q_{T}}{\operatorname{ess} \sup _{i=1}} \sum_{i}^{n} b_{i}^{2}(x, t)$. Choosing in (22) $\delta_{0}=a_{0} / 2$ and using the Gronwall lemma, we obtain

$$
\int_{B^{R}}\left[\left|u_{t}^{N}(\tau)\right|^{2}+\sum_{i=1}^{n}\left|u_{x_{i}}^{N}(\tau)\right|^{2}+\left|u^{N}(\tau)\right|^{2}\right] d x+\int_{Q_{\tau}^{R}}\left[\sum_{i=1}^{n}\left|u_{x_{i}}^{N}\right|^{2}+\left|u_{t}^{N}\right|^{p(x)}\right] d x d t \leq C_{1},
$$


where the constant $C_{1}>0$ is independent of $N$ (but depends on $R$ ).

Using Lemma 3.9 [11, p. 865-866], similarly as in [30, p. 89], we conclude that

$$
\left\|u_{t t}^{N}\right\|_{L^{2}\left(0, T ; H^{-1}\left(B^{R}\right)\right)+L^{p^{\prime}(x)}\left(Q_{T}^{R}\right)} \leq C_{2}
$$

where the constant $C_{2}>0$ is independent of $N$. From (23) and Proposition 3, we have

$$
\int_{Q_{\tau}^{R}}\left|c_{1}\left(x, t, u_{t}^{N}\right)\right|^{p^{\prime}(x)} d x d t \leq c_{1}^{0} S_{p}\left(\left\|u_{t}^{N} ; L^{p(x)}\left(Q_{\tau}^{R}\right)\right\|\right) \leq c_{1}^{0} S_{p}\left(C_{3}\left\|u_{t}^{N} ; L^{2}\left(Q_{\tau}^{R}\right)\right\|\right) \leq C_{4},
$$

where the constant $C_{4}>0$ is independent of $N$.

On the basis of (22)-(25) there exists a subsequence of $\left\{u^{N}\right\}_{N \in \mathbb{N}}$ (we call it $\left\{u^{N}\right\}_{N \in \mathbb{N}}$ again) such that

$$
\begin{aligned}
& u^{N} \underset{N \rightarrow \infty}{\longrightarrow} u^{R} \quad * \text {-weakly in } \quad L^{\infty}\left(0, T ; H_{0}^{1}\left(B^{R}\right)\right), \\
& u_{t}^{N} \underset{N \rightarrow \infty}{\longrightarrow} u_{t}^{R} \quad * \text {-weakly in } \quad L^{\infty}\left(0, T ; L^{2}\left(B^{R}\right)\right), \\
& u_{t}^{N} \underset{N \rightarrow \infty}{\longrightarrow} u_{t}^{R} \quad \text { weakly in } L^{2}\left(0, T ; H_{0}^{1}\left(B^{R}\right)\right) \text { and } L^{p(x)}\left(Q_{T}^{R}\right), \\
& u_{t t}^{N} \underset{N \rightarrow \infty}{\longrightarrow} u_{t t}^{R} \quad \text { weakly in } L^{2}\left(0, T ; H^{-1}\left(B^{R}\right)\right)+L^{p^{\prime}(x)}\left(Q_{T}^{R}\right), \\
& c_{1}\left(\cdot, \cdot, u_{t}^{N}\right) \underset{N \rightarrow \infty}{\longrightarrow} \chi^{R} \quad \text { weakly in } L^{p^{\prime}(x)}\left(Q_{T}^{R}\right) .
\end{aligned}
$$

Then by Theorem $5.1\left[28\right.$, p. 70] we obtain $u_{t}^{N} \underset{N \rightarrow \infty}{\longrightarrow} u_{t}^{R}$ strongly in $L^{2}\left(Q_{T}^{R}\right)$ and a.e. in $Q_{T}^{R}$. Thus, $\chi^{R}=c_{1}\left(x, t, u_{t}^{R}\right)$.

Using Lemma $1.2[28$, p. 20], we prove that

$$
u \in C\left([0, T] ; L^{2}\left(B^{R}\right)\right), \quad u_{t} \in C\left([0, T] ; H^{-1}\left(B^{R}\right)+L^{p^{\prime}(x)}\left(B^{R}\right)\right) .
$$

Similarly as in [12], we prove that $u^{R}(0)=u_{0}^{R}, u_{t}^{R}(0)=u_{1}^{R}$, and the function $u^{R}$ is a weak solution to problem (16)-(18).

\section{PROOF OF MAIN THEOREMS}

Now we will prove Theorem 1.

Proof. Let $u^{1}$ and $u^{2}$ be weak solutions to problem (1)-(3), $u \equiv u^{1}-u^{2}$. Taking into account (14), we obtain

$$
-\int_{Q_{\tau}} u_{t} v_{t} \varphi d x d t=\int_{Q_{\tau}} \frac{\mu}{2}\left|u_{t}\right|^{2} \varphi e^{-\mu t} d x d t-\frac{1}{2} \int_{\mathbb{R}^{n}}\left|u_{t}(\tau)\right|^{2} \varphi e^{-\mu \tau} d x,
$$

where $v=u_{t} e^{-\mu t}$ and $\mu>0$. Then, from the equality of type (8), we get

$$
\begin{gathered}
\frac{1}{2} \int_{\mathbb{R}^{n}}\left|u_{t}(\tau)\right|^{2} \varphi e^{-\mu \tau} d x+\int_{Q_{\tau}}\left[\frac{\mu}{2}\left|u_{t}\right|^{2} \varphi e^{-\mu t}+\sum_{i, j=1}^{n} a_{i j} u_{x_{i}} t\left(u_{t} \varphi\right)_{x_{j}} e^{-\mu t}+\sum_{i, j=1}^{n} b_{i j} u_{x_{i}}\left(u_{t} \varphi\right)_{x_{j}} e^{-\mu t}\right. \\
\left.+\sum_{i=1}^{n} b_{i} u_{x_{i}} u_{t} \varphi e^{-\mu t}+\left(c_{1}\left(x, t, u_{t}^{1}\right)-c_{1}\left(x, t, u_{t}^{2}\right)\right) u_{t} \varphi e^{-\mu t}+c_{2} u u_{t} \varphi e^{-\mu t}\right] d x d t=0 .
\end{gathered}
$$


Now let us make some transformations and let us obtain the required estimates. Then, taking into account conditions of Theorem 1, using (15) and Lemma 1, we get

$$
\begin{aligned}
& \int_{Q_{\tau}} \sum_{i, j=1}^{n} a_{i j} u_{x_{i}} t u_{x_{j} t} \varphi e^{-\mu t} d x d t \geq a_{0} \int_{Q_{\tau}} \sum_{i=1}^{n}\left|u_{x_{i} t}\right|^{2} \varphi e^{-\mu t} d x d t \\
& \int_{Q_{\tau}} \sum_{i, j=1}^{n} a_{i j} u_{x_{i}} u_{t} \varphi_{x_{j}} e^{-\mu t} d x d t \leq \delta_{1} \int_{Q_{\tau}} \sum_{i, j=1}^{n}\left|u_{x_{i}}\right|^{2} \varphi e^{-\mu t} d x d t+\Upsilon_{2}\left(\delta_{1}\right) \int_{Q_{\tau}} \sum_{i, j=1}^{n} a_{i j}^{2}\left|u_{t}\right|^{2} \frac{\left|\varphi_{x_{j}}\right|^{2}}{\varphi} e^{-\mu t} d x d t \\
& \leq n \delta_{1} \int_{Q_{\tau}} \sum_{i=1}^{n}\left|u_{x_{i}}\right|^{2} \varphi e^{-\mu t} d x d t+n^{2}(R+\varkappa)^{2 \theta}\left(\frac{\gamma \widehat{h} \alpha}{\varkappa}\right)^{2} \frac{1}{4 \delta_{1}} \int_{Q_{\tau}}\left|u_{t}\right|^{2}\left(h_{R, \varkappa}\right)^{\gamma-2} e^{-\mu t} d x d t, \quad \delta_{1}>0 ; \\
& \int_{Q_{\tau}} \sum_{i, j=1}^{n} b_{i j} u_{x_{i}} u_{t} \varphi_{x_{j}} e^{-\mu t} d x d t \leq n \delta_{2} \int_{Q_{\tau}} \sum_{i=1}^{n}\left|u_{x_{i}}\right|^{2} \varphi e^{-\mu t} d x d t \\
& +\left(\frac{\gamma \widehat{h} \beta}{\varkappa}\right)^{2} \frac{n^{2}(R+\varkappa)^{2 \theta}}{4 \delta_{2}} \int_{Q_{\tau}}\left|u_{t}\right|^{2}\left(h_{R, \varkappa}\right)^{\gamma-2} e^{-\mu t} d x d t, \quad \delta_{2}>0 \\
& \int_{Q_{\tau}} \sum_{i, j=1}^{n} b_{i j} u_{x_{i}} u_{x_{j} t} \varphi e^{-\mu t} d x d t \geq \frac{b_{0}}{2} \int_{\mathbb{R}^{n}} \sum_{i=1}^{n}\left|u_{x_{i}}\right|^{2} \varphi e^{-\mu \tau} d x+\frac{\mu b_{0}}{2} \int_{Q_{\tau}} \sum_{i=1}^{n}\left|u_{x_{i}}\right|^{2} \varphi e^{-\mu t} d x d t ; \\
& \int_{Q_{\tau}} \sum_{i=1}^{n} b_{i} u_{x_{i}} u_{t} \varphi e^{-\mu t} d x d t \leq \widetilde{B} \delta_{3} \int_{Q_{\tau}} \sum_{i=1}^{n}\left|u_{x_{i}}\right|^{2} \varphi e^{-\mu t} d x d t+\frac{1}{4 \delta_{3}} \int_{Q_{\tau}}\left|u_{t}\right|^{2} \varphi e^{-\mu t} d x d t, \quad \delta_{3}>0 \\
& \int_{Q_{\tau}} c_{2} u u_{t} \varphi e^{-\mu t} d x d t \geq \frac{c_{2,0}}{2} \int_{\mathbb{R}^{n}}|u|^{2} \varphi e^{-\mu \tau} d x+\left(\frac{\mu}{2}-\frac{\left(c_{2, t}\right)^{0}}{2}\right) \int_{Q_{\tau}}|u|^{2} \varphi e^{-\mu t} d x d t .
\end{aligned}
$$

Moreover, $\left(c_{1}\left(x, t, u_{t}^{1}\right)-c_{1}\left(x, t, u_{t}^{2}\right)\right) u_{t} \geq 0$. Thus, from equality (26) we obtain

$$
\begin{gathered}
\int_{\mathbb{R}^{n}}\left[\left|u_{t}\right|^{2}+b_{0} \sum_{i=1}^{n}\left|u_{x_{i}}\right|^{2}+c_{2,0}|u|^{2}\right] \varphi e^{-\mu \tau} d x+\int_{Q_{\tau}}\left[\left(\mu-\frac{1}{2 \delta_{3}}\right)\left|u_{t}\right|^{2}+\left(2 a_{0}-2 n \delta_{1}\right) \sum_{i=1}^{n}\left|u_{x_{i}}\right|^{2}\right. \\
\left.+\left(\mu b_{0}-2 n \delta_{2}-2 \widetilde{B} \delta_{3}\right) \sum_{i=1}^{n}\left|u_{x_{i}}\right|^{2}+\left(\mu-\left(c_{2, t}\right)^{0}\right)|u|^{2}\right] \varphi e^{-\mu t} d x d t \\
\leq \int_{Q_{\tau}}\left[\left(\frac{\gamma \widehat{h} \alpha}{\varkappa}\right)^{2} \frac{n^{2}(R+\varkappa)^{2 \theta}}{2 \delta_{1}}+\left(\frac{\gamma \widehat{h} \beta}{\varkappa}\right)^{2} \frac{n^{2}(R+\varkappa)^{2 \theta}}{2 \delta_{2}}\right]\left|u_{t}\right|^{2}\left(h_{R, \varkappa}\right)^{\gamma-2} e^{-\mu t} d x d t .
\end{gathered}
$$

Let us set $\mu=\mu_{0}+\mu_{1}$. Choosing $\delta_{1}, \delta_{2}, \delta_{3}>0$ sufficiently small and $\mu_{1}>0$ sufficiently large, from (27), it follows that

$$
\begin{aligned}
& \int_{\mathbb{R}^{n}}\left[\left|u_{t}\right|^{2}+b_{0} \sum_{i=1}^{n}\left|u_{x_{i}}\right|^{2}+c_{2,0}|u|^{2}\right] \varphi e^{-\mu \tau} d x+e^{-\mu_{1} T} \int_{Q_{\tau}}\left[\mu_{0}\left|u_{t}\right|^{2}+a_{0} \sum_{i=1}^{n}\left|u_{x_{i}}\right|^{2}\right. \\
& \left.\quad+\mu_{0} b_{0} \sum_{i=1}^{n}\left|u_{x_{i}}\right|^{2}+\mu_{0}|u|^{2}\right] \varphi e^{-\mu_{0} t} d x d t \leq \frac{C_{0}(R+\varkappa)^{2 \theta}}{\varkappa^{2}} \int_{Q_{\tau}}\left|u_{t}\right|^{2}\left(h_{R, \varkappa}\right)^{\gamma-2} e^{-\mu_{0} t} d x d t,
\end{aligned}
$$


where $C_{0}=\frac{n^{2}(\gamma \widehat{h} \alpha)^{2}}{2 \delta_{1}}+\frac{n^{2}(\gamma \widehat{h} \beta)^{2}}{2 \delta_{2}}$. Denote $C_{0} e^{\mu_{1} T} \equiv \widetilde{C}$. Then taking into account (28) and Lemma 1 $\left(\varphi=1\right.$ if $|x| \leq R,\left|h_{R, \varkappa}\right| \leq 1$ if $x \in \mathbb{R}^{n}, h_{R, \varkappa}=0$ if $\left.|x| \geq R+\varkappa\right)$, we obtain

$$
\int_{Q_{\tau}^{R}}\left|u_{t}\right|^{2} e^{-\mu_{0} t} d x d t \leq \frac{\widetilde{C}(R+\varkappa)^{2 \theta}}{\mu_{0} \varkappa^{2}} \int_{Q_{\tau}^{R+\varkappa}}\left|u_{t}\right|^{2} e^{-\mu_{0} t} d x d t .
$$

Let us divide the interval $[R, R+\varkappa]$ on $k$ parts

$$
\left[R ; R+\frac{\varkappa}{k}\right],\left[R+\frac{\varkappa}{k} ; R+\frac{2 \varkappa}{k}\right], \ldots,\left[R+\frac{(k-1) \varkappa}{k} ; R+\frac{k \varkappa}{k}\right] .
$$

Put $\varkappa=\frac{R}{k}$ and estimate the expression

$$
\frac{\widetilde{C}\left(R+\frac{R}{k}\right)^{2 \theta}}{\mu_{0}\left(\frac{R}{k}\right)^{2}}=\frac{\widetilde{C}(R k+R)^{2 \theta} k^{2}}{\mu_{0} k^{2 \theta} R^{2}} \leq \frac{\widetilde{C}(2 k)^{2 \theta} k^{2(1-\theta)}}{\mu_{0} R^{2(1-\theta)}} \leq \frac{\widetilde{C} 2^{2 \theta} k^{2}}{\mu_{0} R^{2(1-\theta)}} .
$$

Denote $\rho_{\theta}\left(R, \mu_{0}, k\right)=\frac{\widetilde{C} 2^{2 \theta} k^{2}}{\mu_{0} R^{2(1-\theta)}}$. Let us choose $R, \mu_{0}$ and $k$ in such way that the next inequality holds:

$$
\rho_{\theta}\left(R, \mu_{0}, k\right) \leq e^{-1}
$$

For that purpose we put $R=2^{j}, \mu_{0}=\lambda_{0} 2^{2(1-\theta) j}$, and $k=\lambda_{1}\left[2^{2(1-\theta) j}\right]$, where $j, \lambda_{0}, \lambda_{1} \in \mathbb{N}$. We choose the parameters $\lambda_{0}$ and $\lambda_{1}$ such that $\lambda_{1}=\left[b 2^{2(1-\theta)}\right]+2$ and $\lambda_{0} \geq \tilde{C} 2^{2 \theta} \lambda_{1}^{2} e$ (here by [w] we denote the entire part of $w$ ), where $b>0$ is taken from (9), $\theta \in(0,1)$ is taken from (A)-(B). Then estimate (30) is correct. Thus, applying (29) $k$ times, we get

$$
\int_{Q_{\tau}^{R}}\left|u_{t}\right|^{2} e^{-\mu_{0} t} d x d t \leq e^{-k} \int_{Q_{\tau}^{2 R}}\left|u_{t}\right|^{2} e^{-\mu_{0} t} d x d t .
$$

Taking into account the inequality $\left|e^{-\mu_{0} t}\right| \leq 1$ which is correct for every $t \in[0, T]$ and the estimate (9), the right-hand side of the last inequality can be estimated by the expression $a e^{-\zeta}$, where $\xi=k-b(2 R)^{2(1-\theta)}$. Then on the basis of choice $k$ and $R$ we get

$$
\xi=\lambda_{1} 2^{2(1-\theta) j}-b 2^{2(1-\theta)} 2^{2(1-\theta) j} \geq\left[2^{2(1-\theta) j}\right]\left(\lambda_{1}-b 2^{2(1-\theta)}\right) \geq\left[2^{2(1-\theta) j}\right] .
$$

Estimate (32) implies that $\xi \rightarrow+\infty$ as $j \rightarrow+\infty$ (and consequently $R \rightarrow+\infty$ ). Then the righthand side of inequality (31) tends to zero as $R \rightarrow+\infty$. Thus, $u^{1}=u^{2}$ a.e. on $Q_{\tau}^{R}$. Hence, taking into account an arbitrariness of $R$ and $\tau \in(0, T]$, we get the uniqueness of the solution to problem (1)-(3) in the domain $Q_{T}$. Theorem 1 is proved.

Let us prove Theorem 2.

Proof. Let $R=R(k)=2^{k}$, where $k \in \mathbb{N}$. We construct sequence $\left\{u^{k}\right\}_{k \in \mathbb{N}}$, where $u^{k}$ is a weak solution to problem (16)-(18) in the domain $Q_{T}^{R(k)}$ and $k \in \mathbb{N}$. The function $u^{k}$ exists by Theorem 3. Let us extend the function $u^{k}$ by zero on $Q_{T} \backslash Q_{T}^{R(k)}$ and let it be again $u^{k}$. Then for every $k \in \mathbb{N}, \tau \in(0, T]$, and $v \in L^{2}\left(0, T ; H_{0}^{1}\left(\mathbb{R}^{n}\right)\right)$ such that $v_{t} \in L^{2}\left(Q_{T}\right)$, we obtain

$$
\begin{aligned}
& \int_{\mathbb{R}^{n}} u_{t}^{k}(\tau) v(\tau) \varphi d x+\int_{Q_{\tau}}\left[-u_{t}^{k} v_{t} \varphi+\sum_{i, j=1}^{n} a_{i j} u_{x_{i} t}^{k}(v \varphi)_{x_{j}}+\sum_{i, j=1}^{n} b_{i j} u_{x_{i}}^{k}(v \varphi)_{x_{j}}+\sum_{i=1}^{n} b_{i} u_{x_{i}}^{k} v \varphi\right. \\
& \left.+c_{1}\left(x, t, u_{t}^{k}\right) v \varphi+c_{2} u^{k} v \varphi\right] d x d t=\int_{\mathbb{R}^{n}} u_{1}^{k}(x) v(0) \varphi d x+\int_{Q_{\tau}}\left[f_{0} v \varphi+\sum_{i=1}^{n} f_{i}(v \varphi)_{x_{i}}\right] d x d t,
\end{aligned}
$$


where $\varphi$ is taken from (11) with $R=R(k)$ (see proof of Theorem 1). Let us take $\varkappa \in[0, R(k)]$. Then supp $\varphi \subset Q^{R+\varkappa} \subset Q^{R(k)+R(k)}=Q^{2^{k}+2^{k}}=Q^{2^{k+1}}=Q^{R(k+1)}$. Consider (33) first for $u^{k+3}$ and then for $u^{k+2}$. On subtracting the obtained equalities, we take $v=u_{t}^{k+3, k+2} \varphi e^{-\mu t}$, where $\mu>0$ and $u^{k+3, k+2}=u^{k+3}-u^{k+2}$. Then similarly as (26), we get

$$
\begin{aligned}
& \frac{1}{2} \int_{\mathbb{R}^{n}}\left|u_{t}^{k+3, k+2}(\tau)\right|^{2} \varphi e^{-\mu \tau} d x+\int_{Q_{\tau}}\left[\frac{\mu}{2}\left|u_{t}^{k+3, k+2}\right|^{2} \varphi+\sum_{i, j=1}^{n} a_{i j} u_{x_{i} t}^{k+3, k+2}\left(u_{t}^{k+3, k+2} \varphi\right)_{x_{j}}\right. \\
& \quad+\sum_{i, j=1}^{n} b_{i j} u_{x_{i}}^{k+3, k+2}\left(u_{t}^{k+3, k+2} \varphi\right)_{x_{j}}+\sum_{i=1}^{n} b_{i} u_{x_{i}}^{k+3, k+2} u_{t}^{k+3, k+2} \varphi \\
& \left.\quad+\left(c_{1}\left(x, t, u_{t}^{k+3}\right)-c_{1}\left(x, t, u_{t}^{k+2}\right)\right) u_{t}^{k+3, k+2} \varphi+c_{2} u^{k+3, k+2} u_{t}^{k+3, k+2} \varphi\right] e^{-\mu t} d x d t=0 .
\end{aligned}
$$

Now, taking into account (34), similarly as (31), we obtain

$$
\int_{Q_{\tau}^{R(k)}}\left|u_{t}^{k+3, k+2}\right|^{2} e^{-\mu_{0} t} d x d t \leq e^{-s} \int_{Q_{\tau}^{R(k+1)}}\left|u_{t}^{k+3, k+2}\right|^{2} e^{-\mu_{0} t} d x d t,
$$

where $R(k)=2^{k}, s=\lambda_{1}\left[2^{2(1-\theta) k}\right], \lambda_{1}=\left[b 2^{6(1-\theta)}\right]+2, \mu_{0}=\lambda_{0} 2^{2(1-\theta) k}$, and $\lambda_{0} \geq \tilde{C} 2^{2 \theta} \lambda_{1}^{2} e$.

It is clear that for every $k \in \mathbb{N}$ the next equality is correct:

$$
\begin{gathered}
\frac{1}{2} \int_{B^{R(k)}}\left|u_{t}^{k}\right|^{2} e^{-\mu \tau} d x+\int_{Q_{\tau}^{R(k)}}\left[\frac{\mu}{2}\left|u_{t}^{k}\right|^{2}+\sum_{i, j=1}^{n} a_{i j} u_{x_{i}}^{k} u_{x_{j} t}^{k}+\sum_{i, j=1}^{n} b_{i j} u_{x_{i}}^{k} u_{t x_{j}}^{k}\right. \\
\left.+\sum_{i=1}^{n} b_{i} u_{x_{i}}^{k} u_{t}^{k}+c_{1}\left(x, t, u_{t}^{k}\right) u_{t}^{k}+c_{2} u^{k} u_{t}^{k}\right] e^{-\mu t} d x d t \\
=\frac{1}{2} \int_{B^{R(k)}}\left|u_{1}^{R(k)}\right|^{2} d x+\int_{Q_{\tau}^{R(k)}}\left[f_{0}^{R(k)} u_{t}^{k}+\sum_{i=1}^{n} f_{i}^{R(k)} u_{x_{i} t}^{k}\right] e^{-\mu t} d x d t
\end{gathered}
$$

From (36), similarly as (28), we obtain

$$
\begin{aligned}
\int_{B^{R(k)}} & {\left[\left|u_{t}^{k}\right|^{2}+b_{0} \sum_{i=1}^{n}\left|u_{x_{i}}^{k}\right|^{2}+c_{2,0}\left|u^{k}\right|^{2}\right] e^{-\mu \tau} d x+\int_{Q_{\tau}^{R(k)}}\left[\left(\mu_{0}-\varepsilon_{1}\right)\left|u_{t}^{k}\right|^{2}+\left(2 a_{0}-\varepsilon_{2}\right) \sum_{i=1}^{n}\left|u_{x_{i}}^{k}\right|^{2}\right.} \\
+ & \left.\mu_{0} b_{0} \sum_{i=1}^{n}\left|u_{x_{i}}^{k}\right|^{2}+\mu_{0}\left|u^{k}\right|^{2}+2 c_{1,0}\left|u_{t}\right|^{p(x)}\right] e^{-\mu_{0} t} d x d t \\
\leq & \int_{B^{R(k)}}\left[\left|u_{1}^{k}\right|^{2}+\sum_{i, j=1}^{n} b_{i j}(0) u_{0, x_{i}}^{k} u_{0, x_{j}}^{k}+c_{2}^{0}\left|u_{0}^{k}\right|^{2}\right] d x+C\left(\varepsilon_{1}, \varepsilon_{2}\right) \int_{Q_{\tau}^{R(k)}} \sum_{i=0}^{n}\left|f_{i}\right|^{2} e^{-\mu t} d x d t .
\end{aligned}
$$

Since

$$
\int_{B^{R(k)}} \sum_{i, j=1}^{n} b_{i j}(0) u_{0, x_{i}}^{k} u_{0, x_{j}}^{k} d x \leq \beta n(R(k))^{\theta} \int_{B^{R(k)}} \sum_{i=1}^{n}\left|u_{0, x_{i}}\right|^{2} d x
$$

taking into account conditions (A), (B), and (C), from (37), we have

$$
\int_{Q_{\tau}^{R(k)}}\left|u_{t}^{k}\right|^{2} d x d t \leq C_{5}\left[\int_{B^{R(k)}}\left(\left|u_{1}\right|^{2}+n(R(k))^{\theta} \sum_{i=1}^{n}\left|u_{0, x_{i}}\right|^{2}\right) d x+\int_{Q_{\tau}^{R(k)}} \sum_{i=0}^{n}\left|f_{i}\right|^{2} d x d t\right],
$$


where the constant $C_{5}>0$ is independent of $k$.

From (38) and (10), it follows that

$$
\int_{Q_{\tau}^{R(k+1)}}\left|u_{t}^{k+3, k+2}\right|^{2} d x d t \leq 2 \int_{Q_{\tau}^{R(k+1)}}\left(\left|u_{t}^{k+3}\right|^{2}+\left|u_{t}^{k+2}\right|^{2}\right) d x d t \leq 4 a \exp \left(b(R(k+3))^{2(1-\theta)}\right) .
$$

Since $\left|e^{-\mu_{0} t}\right| \leq 1$ for every $t \in[0, T]$ and inequalities (35), (39) hold, for every $\tau \in(0, T]$, we get

$$
\begin{aligned}
\int_{B^{R(k)}} & {\left[\left|u_{t}^{k+3, k+2}(\tau)\right|^{2}+\sum_{i=1}^{n}\left|u_{x_{i}}^{k+3, k+2}(\tau)\right|^{2}\right] d x } \\
& +\int_{Q_{\tau}^{R(k)}}\left[\left|u_{t}^{k+3, k+2}\right|^{2}+\sum_{i=1}^{n}\left|u_{x_{i} t}^{k+3, k+2}\right|^{2}+\sum_{i=1}^{n}\left|u_{x_{i}}^{k+3, k+2}\right|^{2}\right] d x d t \leq C_{6} e^{-v},
\end{aligned}
$$

where $v=s-b|R(k+3)|^{2(1-\theta)}$ and the constant $C_{6}>0$ is independent of $k$. Since the equality $u^{k}(x, t)=u^{k}(x, 0)+\int_{0}^{t} u_{t}^{k}(x, s) d s$ holds, we obtain

$$
\int_{B^{R(k)}}\left|u^{k}(x, \tau)\right|^{s} d x \leq C_{7}\left(\int_{B^{R(k)}}\left|u_{0}^{k}\right|^{s} d x+\int_{Q_{\tau}^{R(k)}}\left|u_{t}^{k}\right|^{s} d x d t\right), \quad \tau \in(0, T],
$$

where $s>1$ and the constant $C_{7}>0$ is independent of $k$. Moreover, for every $\eta>0$, we have

$$
\begin{aligned}
\left|u^{k}(x, t)\right| e^{-\eta t / 2} & \leq\left|u^{k}(x, 0)\right| e^{-\eta t / 2}+e^{-\eta t / 2} \int_{0}^{t}\left|u_{t}^{k}(x, s)\right| d s \\
& \leq\left|u^{k}(x, 0)\right|+\int_{0}^{t}\left|u_{t}^{k}(x, s)\right| e^{-\eta s / 2} d s,
\end{aligned}
$$

and so

$$
\begin{aligned}
\int_{Q_{\tau}^{R(k)}}\left|u^{k}\right|^{2} e^{-\eta t} d x d t & \leq \int_{Q_{\tau}^{R(k)}} 2\left(\left|u^{k}(0)\right|^{2}+t \int_{0}^{t}\left|u_{t}^{k}\right|^{2} e^{-\eta s} d s\right) d x d t \\
& \leq 2 T\left(\int_{B^{R(k)}}\left|u_{0}^{k}\right|^{2} d x+T \int_{Q_{\tau}^{R(k)}}\left|u_{t}^{k}\right|^{2} e^{-\eta t} d x d t\right), \quad \tau \in(0, T] .
\end{aligned}
$$

Using (40), (41), and (42), we obtain

$$
\int_{B^{R(k)}}\left|u^{k+3, k+2}(\tau)\right|^{2} d x+\int_{Q_{\tau}^{R(k)}}\left|u_{t}^{k+3, k+2}\right|^{2} d x d t \leq C_{8} e^{-v},
$$

where the constant $C_{8}$ is independent of $k$. Taking into account a choice of $s$ and $R(k)$, the right side of the estimates (40), (43) tends to zero if $k \rightarrow \infty$.

Since $u^{k+j}-u^{k}=\sum_{i=1}^{j}\left(u^{k+i}-u^{k+i-1}\right)$, inequality (40) implies that $\left\{u^{k}\right\}_{k \in \mathbb{N}}$ is a fundamental sequence in the space $C\left([0, T] ; H_{0}^{1}\left(B^{R}\right)\right) \cap L^{2}\left(0, T ; H_{0}^{1}\left(B^{R}\right)\right)$ and $\left\{u_{t}^{k}\right\}_{k \in \mathbb{N}}$ is a fundamental sequence in the space $C\left([0, T] ; L^{2}\left(B^{R}\right)\right) \cap L^{2}\left(0, T ; H^{1}\left(B^{R}\right)\right)$, where $R \geq 1$ is an arbitrary number. 
Hence we have that there exist a subsequence $\left\{u^{k_{1}}\right\}_{k_{1} \in \mathbb{N}} \subset\left\{u^{k}\right\}_{k \in \mathbb{N}}$ and a function $u^{1}$ such that

$$
\begin{aligned}
& u^{k_{1}} \underset{k_{1} \rightarrow \infty}{\longrightarrow} u^{1} \text { in } \quad C\left([0, T] ; H_{0}^{1}\left(B^{1}\right)\right) \cap L^{2}\left(0, T ; H_{0}^{1}\left(B^{1}\right)\right), \\
& u_{t}^{k_{1}} \underset{k_{1} \rightarrow \infty}{\longrightarrow} u_{t}^{1} \text { in } \quad C\left([0, T] ; L^{2}\left(B^{1}\right)\right) \cap L^{2}\left(0, T ; H_{0}^{1}\left(B^{1}\right)\right) .
\end{aligned}
$$

From the sequence $\left\{u^{k_{1}}\right\}_{k_{1} \in \mathbb{N}}$ we choose the subsequence $\left\{u^{k_{2}}\right\}_{k_{2} \in \mathbb{N}}$ such that

$$
\begin{aligned}
& u^{k_{2}} \underset{k_{2} \rightarrow \infty}{\longrightarrow} u^{2} \text { in } C\left([0, T] ; H_{0}^{1}\left(B^{2}\right)\right) \cap L^{2}\left(0, T ; H_{0}^{1}\left(B^{2}\right)\right), \\
& u_{t}^{k_{2}} \underset{k_{2} \rightarrow \infty}{\longrightarrow} u_{t}^{2} \text { in } C\left([0, T] ; L^{2}\left(B^{2}\right)\right) \cap L^{2}\left(0, T ; H^{1}\left(B^{2}\right)\right),
\end{aligned}
$$

and so on.

Let us form a diagonal sequence $\left\{u^{l_{l}}\right\}_{l \in \mathbb{N}}$. By the our construction, this sequence tends to the function $u^{l}$ in each of the domain $B^{l} \times(0, T)$. Besides that, $u^{l}=u^{y}$ in $B^{y} \times(0, T)$, where $l>y$. Let us put $u(x, t)=u^{l}(x, t)$ for $(x, t) \in B^{l} \times(0, T)$. Then

$$
\begin{aligned}
& u^{l_{l}} \underset{l_{l} \rightarrow \infty}{\longrightarrow} u \text { in } \quad C\left([0, T] ; H_{\mathrm{loc}}^{1}\left(\mathbb{R}^{n}\right)\right) \cap L^{2}\left(0, T ; H_{\mathrm{loc}}^{1}\left(\mathbb{R}^{n}\right)\right), \\
& u_{t}^{l_{l}} \underset{l_{l} \rightarrow \infty}{\longrightarrow} u_{t} \text { in } \quad C\left([0, T] ; L_{\mathrm{loc}}^{2}\left(\mathbb{R}^{n}\right)\right) \cap L^{2}\left(0, T ; H_{\mathrm{loc}}^{1}\left(\mathbb{R}^{n}\right)\right)
\end{aligned}
$$

and, passing to the limit in (33) with $v=w \varphi$, we get that the function $u$ is a weak solution to problem (1)-(3) in the domain $Q_{T}$.

\section{REFERENCES}

[1] Adams R.A. Sobolev spaces. Academic Press, New York, San Francisco, London, 1975.

[2] Antontsev S., Ferreira J. Existence, uniqueness and blowup for hyperbolic equations with nonstandard growth conditions. Nonlinear Anal. 2013, 93, 62-77. doi:10.1016/j.na.2013.07.019

[3] Antontsev S., Shmarev S. Evolution PDEs with nonstandard growth conditions. Existence, uniqueness, localization, blow-up. Atlantis Press, Paris, 2015.

[4] Avci M., Ayazoglu (Mashiyev) R., Cekic B. Existance of solutions for an elliptic equation with nonstandard growth. Internat. J. Pure and Appl. Math. 2013, 86 (1), 131-139. doi:10.12732/ijpam.v86i1.10

[5] Bandaliyev R.A., Guliyev V.S., Mamedov I.G., Sadigov A.B. The optimal control problem in the processes described by the Goursat problem for a hyperbolic equation in variable exponent Sobolev spaces with dominating mixed derivatives. J. Comput. Appl. Math. 2016, 305, 11-17. doi:10.1016/j.cam.2016.03.024

[6] Bokalo M.M. Almost periodic solutions of anisotropic elliptic-parabolic equations with variable exponents of nonlinearity. Electron. J. Differential Equations 2014, 2014 (169), 1-13.

[7] Bokalo M.M., Buhrii O.M., Mashiyev R.A. Unique solvability of initial-boundary-value problems for anisotropic elliptic-parabolic equations with variable exponents of nonlinearity. J. Nonlinear Evol. Equ. Appl. 2014, 2013 (6), 67-87.

[8] Bokalo M.M., Ilnytska O.V. Problems for parabolic equations with variable exponents of nonlinearity and time delay. Appl. Anal. 2017, 96 (7), 1240-1254. doi:doi.org/10.1080/00036811.2016.1183771

[9] Bokalo T., Buhrii O. Some integrating by parts formulas in variable indices of nonlinearity function spaces. Visnyk of the Lviv Univ. Ser. Mech. Math. 2009, 71, 13-26. (in Ukrainian)

[10] Buhrii O.M. Finiteness of time vanishing of the solution of a nonlinear parabolic variational inequality with variable exponent of nonlinearity. Mat. Studii 2005, 24 (2), 167-172. (in Ukrainian) 
[11] Buhrii O., Buhrii N. Integro-differential systems with variable exponents of nonlinearity. Open Math. 2017, 15, 859-883. doi:10.1515/math-2017-0069

[12] Buhrii O., Buhrii N. Nonlocal in time problem for anisotropic parabolic equations with variable exponents of nonlinearities. J. Math. Anal. Appl. 2019, 473, 695-711. doi:10.1016/j.jmaa.2018.12.058

[13] Buhrii O.M., Domans'ka G.P., Protsakh N.P. Initial boundary value problem for nonlinear differential equation of the third order in generalized Sobolev spaces. Visnyk of the Lviv Univ. Ser. Mech. Math. 2005, 64, 44-61. (in Ukrainian)

[14] Buhrii O., Hurnyak I., Pukach P., Kholyavka O. Second order hyperbolic variational inequalities with variable exponent of nonlinearity. Visnyk of the Lviv Univ. Ser. Mech. Math. 2012, 77, 41-53. (in Ukrainian)

[15] Coddington E.A., Levinson N. Theory of ordinary differential equations. Izd. Inostr. Lit., Moscow, 1958.

[16] Diening L., Harjulehto P., Hästö P., Rüžička M. Lebesgue and Sobolev spaces with variable exponents. Heidelberg, Springer, 2011.

[17] Domans'ka G.P., Lavrenyuk S.P., Protsakh N.P. Problem for nonlinear hyperbolic equation of the third order. Visnyk (Herald) of Chernivtsi University. Series Math. 2005, 269, 34-42. (in Ukrainian)

[18] Gajewski H., Gröger K., Zacharias K. Nonlinear operator equations and operator differential equations. Mir, Moscow, 1978. (in Russian)

[19] Gao Y., Gao W. Existence of weak solutions for viscoelastic hyperbolic equations with variable exponents. Bound Value Probl. 2013, 2013 (208), 1-8. doi:10.1186/1687-2770-2013-208

[20] Guo B., Gao W. Blow-up of solutions to quasilinear hyperbolic equations with p-Laplacian and positive initial energy. Comptes Rendus Mécanique 2014, 342 (9), 513-519. doi:10.1016/j.crme.2014.06.001

[21] Haehnle J., Prohl A. Approximation of nonlinear wave equations with nonstandard anisotropic growth conditions. Math. Comp. 2010, 79, 189-208. doi:10.1090/S0025-5718-09-02231-5

[22] Kholyavka O.T. Hyperbolic varionation inequality of the third order with variable exponent of nonlinearity. Ukrainian Math. J. 2014, 766, 580-593. doi:10.1007/s11253-014-0955-2 (translation of Ukrainian Math. J. 2014, 66 (4) 580-593. (in Ukrainian))

[23] Lavrenyuk S., Panat O. The mixed problem for a semilinear hyperbolic equation in generalized Lebesgue spaces. Visnyk of the Lviv Univ. Ser. Mech. Math. 2006, 66, 243-260. (in Ukrainian)

[24] Lavrenyuk S.P., Panat O.T. Mixed problem for nonlinear hyperbolic equation in an unbounded domain. Reports of the National Academy of Sciences of Ukraine. 2007, 1, 12-17. (in Ukrainian)

[25] Lavrenyuk S., Panat O. Adjoint problem for hyperbolic equations of the second and third order. Visnyk of the Lviv Univ. Ser. Mech. Math. 2008, 68, 158-170. (in Ukrainian)

[26] Lavrenyuk S.P., Panat O.T. Some variational inequality of the third order with variable exponent of nonlinearity in unbounded domain. Visnyk of the Odessa Univ. Ser. Math. Mech. 2008, 13 (18), 55-61. (in Ukrainian)

[27] Li X., Guo B., Liao M. Asymptotic stability of solutions to quasilinear hyperbolic equations with variable sources. Comput. Math. Appl. 2020, 79 (4), 1012-1022. doi:10.1016/j.camwa.2019.08.016

[28] Lions J.-L. Some methods of solving non-linear boundary value problems. Mir, Moscow, 1972. (in Russian)

[29] Lions J.-L., Magenes E. Non-homogeneous boundary value problems and applications. Mir, Moscow, 1971. (in Russian)

[30] Lions J.-L., Strauss W.A. Some non-linear evolution equations. Bull. Soc. Math. France 1965, 93, $43-96$.

[31] Messaoudi S.A., Talahmeh A.A., Al-Smail J.H. Nonlinear damped wave equation: Existence and blow-up. Comput. Math. Appl. 2017, 74 (12), 3024-3041. doi:10.1016/j.camwa.2017.07.048

[32] Messaoudi S.A., Talahmeh A.A. On wave equation: review and recent results. Arab. J. Math. (Springer) 2018, 7, 113-145. doi:10.1007/s40065-017-0190-4

[33] Mikhailov V.P. Partial differential equations. Nauka, Moscow, 1983. (in Russian) 
[34] Nechepurenko M. The mixed problem for a nonlinear hyperbolic-parabolic system in an unbounded domain. Visnyk of the Lviv Univ. Ser. Mech. Math. 2009, 71, 156-174.

[35] Oleinik O.A., Radkevich E.V. Method of introducing of a parameter for evolution equations. Russian Math. Surveys. 1978, 33, 7-84. (in Russian)

[36] Panat O.T. About the solving of the mixed problem for nonlinear evolutional equation of the third order. Visnyk of the Chernivtsi Univ. Ser. Math. 2007, 336-337, 142-150. (in Ukrainian)

[37] Panat O. Some properties of the solutions to the evolutions equations of the third order with variable exponents of nonlinearity. Visnyk of the Lviv Univ. Ser. Mech. Math. 2012, 71, 184-190. (in Ukrainian)

[38] Panat O.T., Buhrii O.M. Some properties of the solutions to the hyperbolic equations with variable exponents of nonlinearity. Trans. Natl. Acad. Sci. Azerb. Ser. Phys.-Tech. Math. Sci. 2010, 30 (1), 155-160.

[39] Pinasco J.P. Blow-up for parabolic and hyperbolic problems with variable exponents. Nonlinear Anal. 2009, 71 (3-4), 1094-1099. doi:10.1016/j.na.2008.11.030

[40] Pukach P., Il'kiv V., Vovk M., Slyusarchuk O., Pukach Y., Mylyan Y., Auzinger W. On the Mathematical Model of Nonlinear Vibrations of a Biologically Active Rod with Consideration of the Rheological Factor. CEUR Workshop Proceedings 2019, 2488, 30-42.

[41] Pukach P., Il'kiv V., Nytrebych Z., Vovk M., Pukach P. Modified Asymptotic Method of Studying the Mathematical Model of Nonlinear Oscillations under the Impact of a Moving Environment. Advances in Intelligent systems and Computing IV. 2020, 1080, 78-89. doi:10.1007/978-3-030-33695-0_7

[42] Rădulescu V., Repovš D. Partial differential equations with variable exponents: variational methods and qualitative analysis. CRC Press, Boca Raton, London, New York, 2015.

[43] Rủžička M. Electrorheological fluids: modeling and mathematical theory. Springer-Verl., Berlin, 2000.

Received 11.08.2020

Бугрій О.М., Холявка О.Т., Пукач П.Я., Вовк М.І. Задача Коші для гіперболіиних рівнянь третього порядку зі змінним показником нелінійності // Карпатські матем. публ. - 2020. — Т.12, №2. - С. 419-433.

Аосліджуються слабкі розв'язки задачі Коші для гіперболічних рівнянь третього порядку зі змінним показником нелінійності. Задача вивчається в деяких класах функцій, зокрема, в просторах Аебега зі змінними показниками. Знайдено достатні умови існування та єдиності розглядуваної задачі.

Ключові слова і фрази: нелінійне гіперболічне рівняння, задача Коші, змінний показник нелінійності, простір Аебега зі змінним показником. 